\title{
Exploring gatekeeper mutations in EGFR through
}

\section{computer simulations}

\author{
Srinivasaraghavan Kannan, ${ }^{a^{*}}$ Stephen J Fox ${ }^{a}$, Chandra S. Verma, ${ }^{a b, c^{*}}$
}

${ }^{a}$ Bioinformatics Institute, Agency for Science Technology and Research (A*STAR), 30 Biopolis Street, \#07-01 Matrix, Singapore 138671

${ }^{b}$ School of Biological Sciences, Nanyang Technological University, 60 Nanyang Drive, Singapore 637551

${ }^{c}$ Department of Biological Sciences, National University of Singapore, 14 Science Drive 4, Singapore 117543

${ }^{*}$ Corresponding authors: Srinivasaraghavan Kannan, Bioinformatics Institute (A*STAR), 30, Biopolis Street, 07-01 Matrix, Singapore 138671, Singapore, E-mail: raghavk@bii.astar.edu.sg; Tel: +65 6478 8353; Fax: +65 6478 9048. Chandra S. Verma, Bioinformatics Institute (A*STAR), 30, Biopolis Street, 07-01 Matrix, Singapore 138671, Singapore, E-mail: chandra@bii.a-star.edu.sg; Tel: +65 6478 8273; Fax: +65 64789048.

\section{Methods}

\section{Simulations}

Systems used in this current study are taken from our previous work [1]. Each system was subject to MD simulations which were carried out with the pemed.CUDA module of the program Amber16 [2]. All atom versions of the Amber 14SB force field (ff14SB) [3] and the general Amber force field (GAFF) [4] were used for the protein and the inhibitors respectively. Gefitinib and erlotinib are non-covalently bound while afatinib is covalently linked to Cys 797 of EGFR. Therefore, parameters for afatinib covalently bonded with Cysteine 
were derived using the antechamber module of Amber 16. The Xleap module was used to prepare the system for the MD simulations. All simulation systems were neutralized with appropriate numbers of counter ions. Each neutralized system was solvated in an octahedral box with TIP3P [5] water molecules, leaving at least $10 \AA$ between the solute atoms and the borders of the box. All MD simulations were carried out in explicit solvent at 300K. During the simulations, the long-range electrostatic interactions were treated with the particle mesh Ewald [6] method using a real space cut off distance of $9 \AA$. The Settle [7] algorithm was used to constrain bond vibrations involving hydrogen atoms, which allowed a time step of $2 \mathrm{fs}$ during the simulations. Solvent molecules and counter ions were initially relaxed using energy minimization with restraints on the protein and inhibitor atoms. This was followed by unrestrained energy minimization to remove any steric clashes. Subsequently the system was gradually heated from 0 to $300 \mathrm{~K}$ using MD simulations with positional restraints (force constant: $50 \mathrm{kcal} \mathrm{mol}^{-1} \AA^{-2}$ ) on protein and inhibitors over a period of $0.25 \mathrm{~ns}$ allowing water molecules and ions to move freely. During an additional $0.25 \mathrm{~ns}$, the positional restraints were gradually reduced followed by a 2 ns unrestrained MD simulation to equilibrate all the atoms. All the simulations were carried out in triplicates (three independent MD simulations initiated with different initial velocities) for $250 \mathrm{~ns}$ with conformations saved every $10 \mathrm{ps}$. The systems that we studied are $E G F R^{W T}$ and the 8 mutants $\left(E G F R^{T 790 M}, E G F R^{T 790 L}, E G F R^{T 790 A}\right.$, $E G F R^{T 790 R}, E G F R^{T 790 N}, E G F R^{T 790 S}, E G F R^{T 790 P}, E G F R^{T 790 I}$ ), each bound separately to ATP or to a drug (erlotinib, gefitinib and afatinib).

\section{Binding Free energy calculations}

Relative binding free energies were calculated using the thermodynamic cycle shown below:

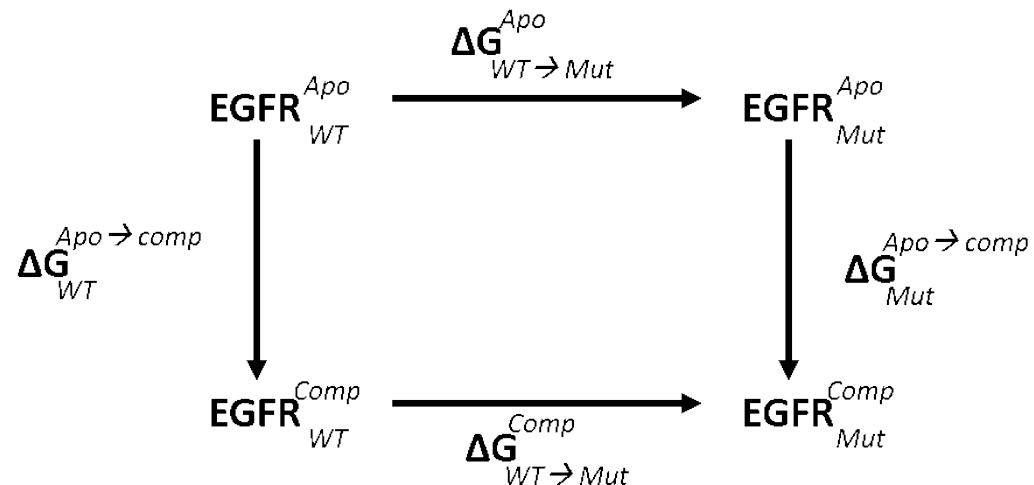


From this cycle, we can calculate the difference in the free energy for the binding of the ligand to the wild type and a mutant.

$$
\begin{aligned}
& \Delta \Delta \mathbf{G}_{\text {bind }}=\Delta \mathbf{G}_{\text {Mut }}^{A p o \rightarrow C o m p}-\Delta \mathbf{G}_{W T}^{A p o \rightarrow C o m p} \\
& \Delta \Delta \mathbf{G}_{\text {bind }}=\Delta \mathbf{G}_{W T \rightarrow \text { Mut }}^{\text {Comp }}-\Delta \mathbf{G}_{W T \rightarrow \text { Mut }}^{A p o}
\end{aligned}
$$

where $\Delta \mathrm{G}$ is the binding free energy of mutating the WT protein, either in its apo state or bound to a ligand.

The $\boldsymbol{\Delta} \mathbf{G}_{\mathbf{W T} \rightarrow \text { Mut }}$ energy differences in the apo and complex forms are calculated using alchemical transformation methods (thermodynamic integration (TI) and free energy perturbation (FEP)). The free energy differences between the WT and mutant proteins were calculated by gradually perturbing from one to the other in a series of discrete steps, represented by $\lambda$ values. The $\lambda$ values vary from 0 to 1 corresponding respectively to the initial $A$ and final B states. Two different transformations needed to be simulated: WT to mutant in the apo state and WT to mutant when both are complexed with a ligand. We use a total of $11 \lambda$ windows $(0.0,0.10,0.20,0.30,0.40,0.50,0.60,0.70,0.80,0.90,1.0)$ for the TI and $19 \lambda$ windows $(0.05$, $0.10,0.15,0.20,0.25,0.30,0.35,0.40,0.45,0.50,0.55,0.60,0.65,0.70,0.75,0.80,0.85,0.90$, 0.95 ) for the FEP/MBAR simulations at $300 \mathrm{~K}$. At each $\lambda$ window we carried out 500 steps of steepest descent minimization, a 50 ps NVT density equilibration run, and a 5 ns NPT production run. The conformation of the mutant residue was taken from the most populated conformation sampled during standard MD simulations (previous paragraph). A time step of 1 fs is used together with the SHAKE algorithm. Ewald sums with a $9 \AA$ cutoff in the real part, isotropic pressure scaling, and a Langevin type thermostat to maintain the temperature at 300 K were also used. To improve convergence, "soft-core" potentials were applied both to the Lennard-Jones and the Coulombic potentials as implemented in AMBER 16 [2]. This makes it possible to perform both charge and van der Waals mutations in the same step and therefore the alchemical transformation was carried out in a single step, referred to as the singletransformation approach (STA). Free energy derivatives $(\partial \mathrm{V} / \partial \lambda)$ were collected independently for each $\lambda$ from the production run. In the TI method, the free energy difference is calculated from the integral of $\partial V(\lambda) / \partial \lambda$ from 0 to 1 , where $V$ is the potential energy. In the case of FEP, 
free energy differences were calculated using the multi Bennett acceptance ratio (MBAR) method as implemented in Amber 16 [2].

1. Kannan, S.; Pradhan, M.R.; Tiwari, G.; Tan, W.C.; Chowbay, B.; Tan, E.H.; Tan, D.S.; Verma, C. Hydration Effects on the Efficacy of the Epidermal Growth Factor Receptor Kinase Inhibitor Afatinib. Scientific Reports. 2017, 7, 1540.

2. Case, D.A.; Ben-Shalom, I.Y.; Brozell, S.R.; Cerutti, D.S.; Cheatham, III, T.E.; Cruzeiro, V.W.D.; Darden, T.A.; Duke, R.E.; Ghoreishi, D.; Gilson, M.K.; Gohlke, H.; Goetz, A.W.; Greene, D.; Harris, R.; Homeyer, N.; Izadi, S.; Kovalenko, A.; Kurtzman, T.; Lee, T.S.; LeGrand, S.; Li, P.; Lin, C. ; Liu, J.; Luchko, T.; Luo, R.; Mermelstein, D.J.; Merz, K.M.; Miao, Y.; Monard, G.; Nguyen, C.; Nguyen, H.; Omelyan, I.; Onufriev, A.; Pan, F.; Qi, R.; Roe, D.R.; Roitberg, A.; Sagui, C.; SchottVerdugo, S.; Shen, J.; Simmerling, C.L.; Smith, J.; Salomon-Ferrer, R.; Swails, J.; Walker, R.C.; Wang, J.; Wei, H.; Wolf, R.M.; Wu, X.; Xiao, L.; York D.M.; Kollman. P.A. AMBER 16, University of California, San Francisco. 2016.

3. Maier, J.A.; Martinez, C.; Kasavajhala, K.; Wickstrom, L.; Hauser, K.E.; Simmerling, C. ff14SB: Improving the Accuracy of Protein Side Chain and Backbone Parameters from ff99SB. J. Chem. Theory Comput. 2015, 11, 3696-3713.

4. Wang, J.; Wolf, R.M.; Caldwell, J.W.; Kollman, P.A.; Case, D.A. Development and Testing of a General Amber Force Field. J. Comput. Chem. 2004, 25, 1157-1174.

5. Jorgensen, W.L.; Chandrasekhar, J.; Madura, J.D.; Impey, R.W.; Klein, M.L. Comparison of simple potential functions for simulating liquid water. J. Chem. Phys. 1983, 79, 926-935.

6. Darden, T.; York, D.; Pedersen, L. Particle mesh Ewald: An N_log(N) method for Ewald sums in large systems. J. Chem. Phys. 1993, 98, 10089-10092.

7. Miyamoto, S.; Kollman, P.A. Settle: An analytical version of the SHAKE and RATTLE algorithm for rigid water models. J. Comput. Chem. 1992, 13, 952-962. 